\title{
EL ENVEJECIMIENTO: OPORTUNIDAD PARA UNA MEDICINA EN BUSCA DE SUS FINALIDADES*
}

\author{
Pierre Boitte**
}

\begin{abstract}
Resumen
El envejecimiento y la atención geriátrica constituyen los dos aspectos (colectivo e individual) de una misma realidad cultural y social que deberían incitar a la medicina a una saludable transformación de su práctica, que la volviera más consciente de sus propios límites y finalidades. Que la volviera también más apta para convencer a la sociedad de admitir el envejecimiento; una sociedad dispuesta, con mucha facilidad, a desprenderse y a encargar a la medicina de tareas sociales y políticas que no son de competencia médica. Esta es la idea central sostenida por este texto.
\end{abstract}

PALABRAS CLAVE: Envejecimiento; atención geriátrica; gerontología; adulto mayor.

\begin{abstract}
Ageing: Opportunity for a medicine in search for goals

Ageing and geriatric care constitute two aspects (collective and individual) of the same social and cultural reality, and should motivate medicine towards a healthy transformation of its practice, therefore becoming more aware of its own limitations and goals. Also becoming more appropriate for convincing society to accept ageing; a society which willingly would delegate medicine certain social and political tasks, even though they are not medical issues. This is the main idea sustained in this text.
\end{abstract}

KEY WORDS: Ageing; geriatric care; gerontology; geriatrics; older people.

Texto traducido al español por el Dr. Sergio Zorrilla F.

* Sociólogo, Filósofo y Doctor en Salud Pública. Profesor Facultés Universitaires de Notre-Dame de la Paix, Belgique; Profesor Investigador Université Catholique de Lille, France y Consultor de Ética de Clinique Saint-Pierre d'Ottignies, Belgique. 


\section{Resumo \\ O envelhecimento: oportunidade para uma medicina em busca de suas finalidades}

O envelhecimento e a atenção geriátrica constituem dois aspectos (coletivo e individual) de uma mesma realidade cultural e social que deveriam estimular a medicina a uma saudável transformação de sua prática, que a faria mais consciente de seus próprios limites e finalidades. De igual modo, a faria mais apta a convencer a sociedade em aceitar o envelhecimento. Imperioso reconhecer que essa sociedade atribui à Medicina encargos de natureza social e política que escapam de sua competência.

É a tese central do presente texto.

PALAVRAS CHAVES: Envelhecimento; atenção geriátrica; gerontología; geriatría; idosos.

\section{Résumé}

Le vieillissement, chance d'une médicine à la recherche de ses finalités

Le vieillissement et sa prise en charge gériatrique constituen les deux faces (collective et individuelle) d'une même realité culturelle et sociale qui devrait inciter la médecine à une salutaire transformation de sa pratique, qui la rendrait plus consciente de ses propres limites et de ses finalités, qui la rendrait aussi plus apte à les faire admettre à une société prompte à se débarrasser sur la médecine de tâches sociales et politiques qui ne relèvent pas de la compétence médicale: telle est l'idée défendue dans ce texte.

MOTS CLÉS: Vieillissement; soins en gériatrie; gérontologie; gériatrie; personnes âgées. 


\section{Una imagen negativa de la vejez marginalizada por la sociedad}

Desde hace treinta años, dada la manera como nuestras sociedades se preocupan del envejecimiento colectivo e individual, se han producido imágenes negativas de este doble fenómeno. Como numerosos sociólogos lo han constatado, las representaciones colectivas sobre la vejez vehiculan la idea de una muerte social, precursora de la muerte biológica $^{i}$. Las imágenes producidas socialmente -que se plantean como muchas otras imágenes posibles de la vejez (desde el «flamante adulto» siempre bien erguido con más de 80 años, al viejo dependiente y/o demente)- dan cuenta sobre los «procesos de diferenciación progresiva, de separación, de estigmatización» ( 1 p.10) generados después que nuestras sociedades tomaron conciencia de su envejecimiento demográfico. De la tercera edad, inventada en los años 70, se pasó, en los años 80 a la cuarta edad, aquella en donde verdaderamente las cosas se degradan, donde la dependencia, las diversas perdidas y el handicap se instalan, cuando no se trata de la demencia llamada «senil», esperando la muerte anunciada. Hoy en día nos encontramos hablando del riesgo de «interrupción voluntaria de la vejez" ( 2 ), denunciando el escándalo de los viejitos «desnutridos, deshidratados, infectados, llenos de escaras, postrados, confundidos», que invaden en Francia los hospitales públicos durante las fiestas de fin de año (3), recolectando testimonios cada vez más numerosos sobre el maltrato de las personas ancianas (4, p. 8) o recordando que ser viejo no es equivalente a estar enfermo o incapaz de aportar algo a la sociedad (5). Estas realidades y discursos, una vez evocados, aparecen poco tranquilizadores respecto de la percepción que tienen nuestras sociedades sobre la vejez.

Para más detalles consultar Boitte (1997)
Las representaciones más corrientes de la vejez (esquemáticamente el «dependiente» $\mathrm{y}$ «el socialmente útil») ponen en juego una visión utilitarista de los viejos: en los dos casos, se les mide en función de lo que pueden aún aportar a la sociedad (los «buenos» viejos que ayudan a la sociedad a través del voluntariado y los «dependientes» que «cuestan», a pesar que su atención puede generar la creación de nuevos empleos). Sin embargo, estas representaciones empobrecen dramáticamente la realidad de las viejas y de los viejos: más del $80 \%$ de estos no son ni dependientes, ni comprometidos con el mundo asociativo. Por ello aparecen como excluidos de las imágenes que circulan sobre la vejez, sin existencia social, ni reconocidos públicamente como una realidad. Es posible identificar «un movimiento colectivo buscando negar la vejez como una etapa especifíca en la trayectoria de vida y, por ese solo hecho, pudiendo existir sin tener que someterse a los imperativos de la economía mercantil y las exigencias de la ideología utilitarista» (1).

De manera coherente con lo dicho, la vejez se ha convertido progresivamente en el terreno de acciones de los profesionales de salud, al menos, respecto de los ancianos cuyo estado requería cuidados de salud: durante los años 80 se desarrollaron políticas de medicalización para hacerse cargo de la vejez. Los expertos, en particular médicos, fueron encargados por la sociedad -lo que aceptaron con más o menos reticencia o malestar- de «gestionar» los problemas específicamente sanitarios de los más viejos y los más dependientes de entre nosotros. A través de esto han contribuido, sin tener conciencia, a eliminar una parte considerable de la dimensión social de la vejez. El viejo se ha convertido, por la vía de la medicina, en un objeto de estudio y en un deber de encargarse, situado en un mundo a parte, al margen, a impedir, por lo tanto, que permanezca- sería necesario decir, a pesar de su edad-, un sujeto ciudadano. Esta si- 
tuación, según lo muestran numerosos estudios, aparece aún como poco aceptable, puesto que en término medio, la salud de la avanzada edad mejora, tanto en términos objetivos como subjetivos. Así, se constata en Francia una evolución de la esperanza de vida de dos meses a dos meses y medio por año hasta la mitad de los años 90 (74 años para los hombres en 1996 y 82 años para las mujeres), una progresión de la esperanza de vida sin gran incapacidad para el decenio 8090 (lo que debe ser aún confirmado para el último decenio), un acceso más tardío al estado de dependencia y una estabilización de la duración de este estado, la continuidad del retroceso de los riesgos de deceso de los sujetos de edad. Siempre en el caso de Francia, el estado de salud de las personas de más de 70 años se consideraba como satisfactorio o muy satisfactorio para el $70 \%$ de ellas (6, p.36). La distancia entre esta realidad sanitaria y la representación que ofrece la sociedad de esta realidad se avizora, por lo tanto, muy problemática.

\section{Dificultades y deficiencias en el hacerse cargo de las personas de edad avanzada}

Paralelamente a esta percepción colectiva negativa de la vejez, se aprecia una dificultad real para los médicos y para el personal de salud en general, de comprender lo que se acostumbra llamar el «paciente geriátrico». No es raro ver, por otra parte, que los cercanos al paciente de edad rechazan la manera como los hospitales se encargan de estas personas: a menudo, por ejemplo, se critica a los equipos su incapacidad de considerar el paciente independientemente de un órgano enfermo. Tampoco es raro escuchar, de parte del personal de salud, el relato de situaciones en que las personas de edad avanzada, aunque también de las personas inconscientes, de las personas dementes o de los enfermos mentales, sufren de falta de respeto manifiesto y negligencias en los cuidados de salud: despersonalización (identificación sistemática a través de expresiones tales como «mamita»o «papito»), infantilización, tuteo sistemático, falta de información, ausencia de respeto de la autonomía, destrucción del espacio íntimo y privado. En ciertos casos, cuando desaparece la esperanza de curación o de mejoría, la mantención de técnicas invasoras, diagnósticas o terapéuticas, es a menudo vivida como una verdadera violencia respecto de la persona enferma. Algunos profesionales no vacilan en hablar del «fracaso en el hacerse cargo del envejecimiento» $(7)^{\mathrm{ii}}$, fracaso en la calidad del encargarse (calidad médica, calidad funcional, calidad relacional), fracaso de la performance tanto en términos de resultados como en los medios empleados; fracaso, finalmente, en la comunicación con el paciente, pero también con la familia, con su médico de familia y, eventualmente, con el personal de la casa de reposo.

Se pueden, ciertamente, encontrar razones médicas para explicar este fracaso: el carácter múltiple de la patología del adulto mayor, que choca con las especializaciones de punta; la dificultad y la lentitud de un diagnóstico frente a alteraciones de la vigilancia, de la memoria, de la vista; el malestar del examen físico a causa de una cooperación limitada y de una difícil movilización. La frontera entre el examen normal y el examen patológico puede manifestarse en tales circunstancias como extremadamente tenue. La historia de la persona puede igualmente jugar un papel: por ejemplo, un paciente puede vivir su hospitalización como un abandono y entregarse mansamente a su suerte, lo que provoca una rápida degradación de su estado general.

La medicina, el hospital, los profesionales de salud son parte de una sociedad y la negación social de la vejez constituye una

ii Retomo en los dos parágrafos siguientes la argumentación de este texto. 
razón convincente desde el punto de vista del fracaso, tanto como las razones médicas o personales. En el medio hospitalario es difícil considerar el envejecimiento como si no se tratara de una enfermedad, puesto que la sociedad confía a la medicina la misión de prolongar la vida. Al contrario, la presión social sobre el hospital se legitima, a menudo, por la existencia de insuficiencias estructurales en el hacerse cargo -desde un punto de vista social- de las personas de edad mayor, consecuencia lógica de la negación: la imbricación de lo médico y lo social instaura el riesgo de volver tirantes las relaciones entre los terapeutas, la familia y la persona de edad mayor, más aún cuando el hospital, espacio de cuidados de salud, es, además, considerado como un lugar de colocación o como el último recurso frente a una situación de precariedad social.

Para intentar comprender la noción de «fracaso» en el ámbito geriátrico es posible identificar factores psicológicos individuales, factores estructurales y factores de dependencia. Los factores psicológicos derivan, a menudo, del miedo a la muerte, lo que provoca reacciones de fuga o de negación. Los factores estructurales reenvían a varios elementos: la valorización del acto técnico, que explica que a veces los terapeutas se preocupan más de la prescripción médica que de los cuidados de confort; la sobrecarga del trabajo de las enfermeras, que provoca una desmotivación puede también originarse en la carga emocional que constituyen los cuidados de ciertas personas; finalmente, la incompetencia, que no es difícil comprobar en ciertas casas de reposo y de cuidados de salud, como consecuencia de una carencia en la formación del personal.

Los factores de dependencia, finalmente, explicitan la relación asimétrica entre terapeuta-enfermo. El enfermo es un sujeto dependiente de los encargados de los cuidados, quienes se encuentran en posición de fuerza, de autoridad, a menudo con buena salud. La tentación puede surgir en los terapeutas de creer que son más competentes que los mismo enfermos, para conocer sus necesidades. Esta tentación será tanto mayor que el anciano podrá ser considerado por la propia sociedad como una parte desdeñable.

El conjunto de estos factores puede generar verdaderas «desviaciones» en algunos profesionales de salud, incluso en el seno de todo un servicio hospitalario, donde «la degradación progresiva de la calidad en los cuidados termina por no escandalizar a nadie y (donde) la ley del silencio se instaura» (8).

\section{Preguntas a la práctica médica}

El aumento creciente del número de ancianos en el hospital plantea numerosas preguntas a la práctica médica. ¿Qué cuidados procurar a las personas de edad avanzada? Evidentemente de cuidados de calidad, pero, ¿Según qué criterios? ¿Cuáles antibióticos para un viejo demente? ¿Cuánto tiempo? ¿Hasta dónde proseguir una eventual escalada? ¿Hasta la reanimación? ¿Y luego, cómo no obstinarse, en la medida de lo posible? ¿Cómo comunicarse con personas a veces confusas? Nuestra intención no es de abordar detalladamente cada una de estas preguntas, sino que colocar el acento sobre un cierto funcionamiento de la medicina contemporánea en el hacerse cargo del envejecimiento en el hospital. Cuatro preguntas retendrán nuestra atención.

\section{La insistencia o la abstención terapéutica}

El carácter superfluo o inútil de ciertas prácticas al final de la vida pueden ocasionar sufrimientos en los pacientes y los terapeutas pueden considerar éstas como desprovistas de sentido, lo que no ayuda a los enfermos a soportar estas técnicas. En geriatría, más que en otras prácticas, el riesgo de obstinación y su consecuencia, el abandono terapéutico, se manifiesta siempre en un momento u otro del cuidado de una persona en edad avanzada. 
Este riesgo, de forma más particular, puede traducirse en un encarnizamiento diagnóstico, cuando se prescriben, por ejemplo, exámenes dolorosos e invasivos. ¿Será entonces necesario abstenerse, sabiendo que esta abstención diagnóstica volverá difícil la opción de una actitud terapéutica eventualmente benéfica para el paciente? La pregunta se complica doblemente. Por un lado, la deontología médica estipula al mismo tiempo que «el médico debe siempre elaborar su diagnóstico apoyándose, en la medida de lo posible, en los métodos científicos mejor adaptados» y que este mismo médico debe «evitar toda obstinación irrazonable en las investigaciones o en la terapia» (9). Por otra parte, no es extraño «ver pacientes alterados <salir> varias veces de episodios muy graves» (9). Es difícil entonces para un equipo perder la esperanza de que una persona de mucha edad supere una situación difícil. Sin embargo, ¿Nos encontramos por ello en una situación de encarnizamiento terapéutico? Se comprende aquí claramente que las situaciones singulares deben ser evaluadas caso a caso.

Es sin duda más fácil aceptar la decisión de un paciente consciente si este participa y se aferra a terapias apremiantes que encontrarse, por ejemplo, en una situación similar con un paciente aquejado de demencia durante largo tiempo. Esta última situación debe ser objeto de una evaluación muy seria a fin de debatir prioritariamente la temática de la calidad de vida. Parece seguro que «buscar sólo el objetivo de prolongar la vida, mientras que el deterioro progresivo y crítico de los principales sistemas funcionales parece conducir a una muerte inevitable, no es un objetivo independiente y primordial de la medicina» (10). Se trata, por lo tanto, de evitar en estas situaciones el paso al acto que constituye a menudo el encarnizamiento terapéutico, como consecuencia de la ausencia de reflexión y discusión en común del equipo de médicos y de los terapeutas en un sentido amplio.
En todo caso, persiste el problema de determinar en qué consisten los cuidados médicamente apropiados en tales situaciones, sabiendo, por otra parte, que el hospital no es el único lugar donde las personas pueden sufrir un eventual encarnizamiento; la cuestión se plantea también para las casas de reposo y, eventualmente, para las instituciones psiquiátricas. Sin duda la reflexión podría determinar mínimamente que «abstenerse de utilizar medios desproporcionados o inútiles en ciertas situaciones resulta simplemente de una medicina adaptada al estado y a las necesidades del paciente, a fin de respetar su muerte cuando no se puede más respetar la vida» (11).

\section{La ausencia de consentimiento y el rechazo de cuidados}

Respecto de la ausencia de consentimiento es innegable que se trata de una práctica mucho más corriente hacia los ancianos que hacia otros enfermos, incluso si se considera que en Europa Central el médico tiene la obligación de obtener el consentimiento del enfermo, antes de procurarle cualquier cuidado, tratamiento o investigación, así éste sea un anciano. Aún se ven actitudes inaceptables, buscando acallar o desatender a las personas de mucha edad, porque tienden a olvidar o no comprenden bien.

¿Cómo se puede comprender dicha situación? Dos razones coexisten. Primeramente, «toma tiempo informar a un enfermo, asegurarse de su buena comprensión, escuchar su respuesta, tomar en cuenta sus reservas o miedos, negociar con él. Es necesario también tener mucha paciencia cuando la persona es sorda o lenta para integrar las informaciones» (12). Es probablemente difícil exigir tal actitud de un médico o de una enfermera cuando el personal es, a veces, poco numeroso y se encuentra sobrecargado. Pero más fundamentalmente, «para algunos terapeutas o cercanos, la persona de edad es incapaz de dar una opinión, su edad parece 
arrebatarle su estatuto de adulto. A menudo es menos escuchado que un niño. Los otros deciden por ellos!» (12).

En cuanto al rechazo de cuidados, es a menudo percibido de manera tan negativa por los equipos de terapeutas, que desestabiliza su voluntad por hacer lo mejor para el paciente que les ha sido confiado. La acción de cuidados se ve perturbada y nos es raro que se produzca un conflicto relacional con el paciente. Efectivamente, puede sentirse como profundamente perturbador ver ir hacia la muerte a un paciente que, por ejemplo, habría rechazado entrar en reanimación. El rechazo, en la medida que refleja adecuadamente la manera como el paciente percibe el cuidado que se le ha propuesto, incluye también a aquel que lo propone. Por esto, en alguna medida, el fracaso de la comunicación con este paciente es también el fracaso de la negociación mantenida con él. El rechazo, siempre posible, explicita la necesaria vigilancia en toda presentación y explicación de un cuidado propuesto por el terapeuta. Este rechazo manifiesta igualmente la autonomía del enfermo, de este otro humano siempre capaz «de desactivar todas las estrategias, las seducciones, cualquiera sean los ideales más sublimes y los proyectos de cuidado más elaborados» (13). Un comienzo de solución está marcado por el diálogo y la capacidad de negociar la presencia de los respectivos proyectos (proyecto de cuidados y proyecto de vida) para intentar encontrar un compromiso. Tal diálogo, si es posible, puede permitir la clarificación sobre el contenido del rechazo: ¿se trata de un rechazo del mundo de los terapeutas (por un paciente, por ejemplo, cuya intimidad sería violada de manera permanente)? ¿se trata del rechazo de cuidados inapropiados? En caso de fracaso de este diálogo el equipo debería ser capaz de aceptar sus propios límites y renunciar a una voluntad de dominio sobre una vida que no le pertenece.
Evidentemente, para el terapeuta aceptar este rechazo es equivalente a rehusar practicar un cuidado que juzga necesario, lo que no puede acontecer sin conflicto para su conciencia. Pero ignorar este rechazo es equivalente a rechazar el derecho al consentimiento del paciente. ¿En nombre de qué valores sería posible actuar por el bien del paciente, pero en contra de su voluntad? ¿Dónde se encuentra la frontera entre una práctica eventualmente justificable y el maltrato al cual hacíamos referencia? Por otro lado, convencer, persuadir y negociar una parte del cuidado propuesto no es siempre posible en función de las circunstancias.

\section{El sufrimiento de los terapeutas en geriatría}

Vale la pena abordar aquí una tercera interrogante que concierne al sufrimiento de los terapeutas. Numerosos otros servicios hospitalarios pueden ser tocados por este fenómeno: todas las «patologías tristes», aquellas de las cuales es difícil sanar, como en oncología, neurología, psiquiatría a veces, enfermedades infecciosas. En geriatría, más que en otra parte, «el enfrentamiento cotidiano con la vejez, el deterioro físico y psíquico y la muerte, es difícil» (12). Las ocasiones no faltan para someter a prueba las emociones y los sentimientos. Puede dar la impresión de cierta impotencia, incluso de ser un mal prestador de cuidados de salud. La falta de atención o, a veces, de recursos, la ausencia de un proyecto y de una vida de equipo (donde espacios de palabra o de apoyo psicológico sean ofrecidos a los terapeutas) puede desembocar en un verdadero malestar existencial y/o en situaciones de cansancio y usura psíquica. La multiplicación de tales contextos de cuidados, donde el terapeuta no tiene la ocasión de gratificarse con la sanación del enfermo, como consecuencia del abandono de éste del hospital o por el evitamiento de la muerte, debería compro- 
meter a la medicina a reflexionar, un poco más de lo que lo hace actualmente, sobre el sentido de ciertas prácticas de salud.

\section{Las peticiones de eutanasia}

Situaciones desesperadas de sufrimiento provocadas por un encarnizamiento terapéutico pueden desembocar en la petición, por parte del enfermo, su familia o los terapeutas, de un acto de eutanasia, concebido como la única solución posible para salir de una situación insoportable para todos. El contexto en el cual trabajan los equipos puede permitir a veces, comprender sin necesariamente admitirlo, que los terapeutas se sientan tentados por tal solución, y en ciertas circunstancias -como suele ocurrir actualmente- pasen al acto. Sin entrar en el debate contemporáneo sobre la eutanasia y en relación con las solicitudes de los cercanos o de los terapeutas, es necesario recordar que «el advenimiento de la muerte le pertenece a cada anciano» (11). Cada uno puede tener buenas razones de tomarse el tiempo de morir, imprevisibles para los terapeutas. Además, un mejoramiento inesperado del estado de consciencia puede siempre acontecer en los días y horas antes de la muerte, lo que permite, a veces, intercambios de último momento. En este sentido el equipo de terapeutas «no tiene el derecho de anticipar el momento de la muerte de los pacientes» (11). En lo que atañe a las demandas emitidas por el enfermo, el debate actual muestra que el hecho de escuchar atentamente sus peticiones y tener un diálogo respecto de ellas hace que los cuidados paliativos o un acompañamiento hacia el final de la vida reduzcan estas demandas en aproximativamente un $1 \%$ de los fines de vida en medio hospitalario. Solamente respecto de estas situaciones, excepcionales, a menudo extremas, es posible que la consciencia humana sea conducida al punto más radical de su compromiso y que la transgresión de la prohibición del homicidio pueda parecer legítima. Sin embargo, el verdadero problema, parece más bien involucrar la existencia-respecto del final de la vida, tanto en el hospital como en el domicilio- de prácticas clandestinas y expeditivas de eutanasia, constatadas por muchos terapeutas. Frente a tales situaciones toda persona al final de la vida debería poder contar, de forma prioritaria, con el compromiso solidario del médico, de los profesionales de la salud y de la sociedad cualesquiera sean las condiciones de su existencia- antes de asegurarse que se escuchará de forma prioritaria su petición de terminar con la vida.

\section{Interrogantes sobre las finalidades de la medicina}

Las preguntas planteadas a la práctica médica en materia de geriatría incitan a interrogarse de manera cada vez más fundamental sobre los objetivos de la medicina y sobre los medios invertidos en su realización.

\section{Medicalización de la vida y politización de la medicina}

Es posible determinar esquemáticamente tres objetivos para la medicina actual: o bien la medicina cura las enfermedades de los individuos, o bien mejora, en una concepción holística, la salud global (psíquica y mental) de los individuos o, finalmente, se preocupa de elevar el nivel general de la salud pública. La tarea médica, pues, ¿se limita a combatir las enfermedades (objetivo tradicional de la medicina que consiste en trabajar con el cuerpo para obtener una mejor salud)? ¿o este objetivo llega hasta la preocupación de la capacidad física aunque también mental de los pacientes a fin de realizar sus objetivos de vida (concepción holística)? Se agrega entonces a la búsqueda de la curación, la prevención, la educación médica, las terapias mentales, la readaptación del paciente a las condiciones sociales «normales». Nos podemos referir también a la salud de una población: en este caso, la curación de los 
individuos es sólo un objetivo intermediario al servicio del objetivo más global del estado de salud de una colectividad. Aquí los sectores sociales, educativos, políticos y legislativos pueden ser integrados al campo de la tarea médica.

A cada uno de estos tres objetivos corresponde un tipo de medios utilizados por la medicina. Para el primero, el cuerpo humano permanece como la fuente esencial de la medicina. Para el segundo, se agrega la dimensión psíquica como medio o como recurso posible. Para el tercero, los medios sociales necesarios para acrecentar la salud son parte integrante del trabajo médico.

Sobre esta base es posible afirmar que mientras más los objetivos de la medicina son definidos por los actores médicos, más los problemas sociales son parte integrante de la atención médica y de la terapia médica y más una medicalización de la sociedad está en curso (en el sentido que el modelo tecnocientífico de la medicina interviene en el diagnóstico y en la «resolución» de problemas sociales cada vez más numerosos). Mientras más los medios de la medicina, reclamados por la medicina y/o procurados por la sociedad, son tomados en consideración, más las áreas de competencia social son definidas desde el exterior de la medicina en relación con una potencial medicalización y más una politización de la medicina es posible (en el sentido en que las políticas públicas se sirven de la medicina para justificar sus objetivos). Es importante, además, observar que objetivos y medios se refuerzan y que en la medida que la medicalización de la sociedad avanza, la politización de la medicina se vuelve probable. Basta con observar, por ejemplo, cómo el debate actual sobre la eutanasia es el signo de una medicalización extrema sobre el final de la vida y supone siempre el riesgo de generar una imbricación creciente de los dominios médicos y políticos. La edad mayor constituye igualmente una buena ilustración de este doble movimiento de medicalización de la sociedad (y de la vida) y de politización de la medicina (y de la salud). La medicina ha desarrollado ampliamente sus competencias y capacidades sobre el final de la vida, al punto de haber creado en algunos decenios, al menos, tres especialidades suplementarias: la geriatría, los cuidados paliativos y la medicina del dolor, con la consecuencia de la medicalización al final de la vida. Al mismo tiempo la sociedad -por la vía de los poderes públicos y de las políticas, adecuadamente nombradas «médico-sociales» de la vejezconfiaba casi exclusivamente a la medicina la tarea de encargarse de la salud de los más viejos, sin verdaderamente tomar en cuenta, por ejemplo, las lecciones que se desprenden de las perspectivas epidemiológicas o de la salud pública respecto de la salud de los más viejos.

Con razón podemos interrogarnos sobre el territorio legítimo de la medicina y sobre los límites de la medicalización actual de la vida y de la sociedad, puesto que este doble movimiento de medicalización y politización parece cuestionable. La medicina no tiene el monopolio de la promoción de la salud de los más ancianos (crítica de la medicalización) y existen numerosos medios no médicos de preocuparse de su salud (crítica de la politización). En esta perspectiva crítica «las profesiones médicas en vez de reclamar más poder político deberían subrayar la importancia de su competencia para combatir las enfermedades y para mejorar la salud; al mismo tiempo, deberían claramente afirmar su rol limitado, lo que no impide en nada otros factores y otras maneras de promocionar el mismo objetivo de mejorar la salud» (14). De esta manera, en una perspectiva normativa, los medios de la medicina no deben ser ni demasiados reducidos (la persona enferma no se resume a su enfermedad orgánica), ni demasiados amplios (es necesario saber aceptar, como médico o trabajador de salud, que otros expertos u otras competencias intervengan). De la misma manera, el objetivo de la medicina 
no debe ser ni demasiado limitado (la salud global del paciente a fin de considerar la persona como un todo, en el tratamiento de la enfermedad) ni demasiado amplio (los objetivos de la salud pública no vinculan directamente al médico con su práctica cotidiana, donde importa mucho más que el paciente sienta que el médico se interesa por sus problemas de salud). No obstante, tal perspectiva permanece ampliamente programática y de poca utilidad para la reflexión.

\section{La crisis de las finalidades tradicionales de la medicina}

Avancemos un poco más. El envejecimiento constituye uno de los reveladores de la crisis que afecta a la práctica médica en sus finalidades, al servicio de las cuales son desplegados objetivos y medios. De hecho, las finalidades tradicionales de la medicina son ampliamente fragilizadas. ¿Salvar y prolongar la vida implica que la medicina debe volverse enemiga del envejecimiento y la muerte? ¿Promover y mantener la salud implica que la enfermedad debe ser siempre combatida, cualquiera sea el precio para la persona enferma, a menudo anciana? Calmar el dolor y probablemente el sufrimiento ¿obliga al médico a la eutanasia o al suicidio asistido? ¿conduce a la supresión, casi obligatoria, de toda angustia a través de la prescripción de psicotropos?

La medicalización de amplios sectores de la vida humana crea una gran incertidumbre a propósito de la naturaleza y del alcance de la medicina contemporánea. Esta incertidumbre influye en particular en la práctica clínica, percibida a menudo como confusa, «fracasando en encontrar un buen equilibrio entre el cuidado técnico y el cuidado de confort, entre la victoria sobre la enfermedad y el mejoramiento de la calidad de la vida, entre la reducción de la mortalidad y aquella de la morbilidad» (15). De nuevo el envejecimiento ilustra esta constatación, lo hemos visto en las cuestiones que plantea a la práctica médica.

\section{La preocupación predominante por querer curar}

¿Cómo comprender esta evolución? Sin duda ampliando el cuadro de la reflexión y tratando de comprender las razones de la medicalización de la sociedad. Una hipótesis probable se relaciona con la importancia que nuestras sociedades conceden a la salud, en desmedro de una significación social de la enfermedad, que, por lo demás, ha tenido en todas las sociedades. Así, «nuestra cultura nos enseña a vivir la enfermedad como un sin sentido radical ('lo absurdo' 'el azar') que no revela nada y que nada puede justificar. Esta negación de la experiencia patológica se acompaña de una 'positivación' semántica y de una absolutización de la medicina, la expropiación del sentido esencial de la enfermedad es, en los hechos, el reverso de la expropiación de esta última por el saber médico positivo» (16). Resulta una valorización casi desmesurada de la salud. La cultura médica, en una sociedad que busca sus referencias, propone un sentido: «el sentido médico (...) que consiste en vivir utópicamente para evitar la enfermedad y retrasar la hora de la muerte... para conservar a todo precio el más valioso de los bienes: la salud» (16). La enfermedad sólo se puede concebir en relación con la medicina, que valoriza la salud por sí misma, que no vacila, a veces, en proponer el espejismo de un futuro sin enfermedad mortal-y, ¿por qué no?-, sin envejecimiento. Por ello no es necesario sorprenderse demasiado que la medicina olvide progresivamente lo que la hace existir: responder a la exigencia de la enfermedad humana y, al mismo tiempo, a sus finalidades tradicionales. Así se puede sustentar que «hoy en día, en todas partes, es imperioso... la preocupación por sanar. No es 
sólo el hospital moderno, sino que el conjunto de la sociedad que merece ser definida como 'máquina de curar' a través de esta imagen ideal que se procura a sí misma» (17). La existencia en su totalidad está medicalizada desde el comienzo de la vida a la muerte.

Es posible comprender la perplejidad de los médicos frente a la alteración general de un estado de salud hacia el fin de vida, que no constituye verdaderamente una enfermedad; es incluso posible comprender la tendencia a considerar este estado como mórbido ya que instantáneamente se colocaría al paciente en el campo de la intervención supuestamente eficaz de la biomedicina. Pero, transformar en enfermedad lo que es desgaste y usura general con la esperanza de eliminarla puede generar efectos perversos: aquello que puede provocar sobre la salud ya fragilizada de una persona la relativa incongruencia de la intervención médica (en términos de efectos iatrogénicos, por ejemplo); pero, también, los provocados por la atribución arbitraria del término enfermedad, separando una persona de sus redes relacionales habituales, por la prescripción de una estadía hospitalaria.

\section{El envejecimiento: una suerte para la medicina}

La medicina, aunque lo quisiera, no puede jugar sola el papel de autorregulación de la sociedad en materia de envejecimiento. Éste podría convertirse en una excelente ocasión para la medicina de replantearse finalmente la cuestión sobre la curación, de tomar esta curación siempre implícita como objeto de reflexión, puesto que para el anciano no hay nada más que curar. La muerte tendrá siempre la última palabra («muerte, cuya última palabra es el silencio») (17): ¿Por qué no actuar de tal manera que desde el interior mismo de la medicina esta ambición de curar deje de funcionar, si no como supe- ración de la negación de la muerte, a lo menos como desafío frente a esta violencia de la muerte siempre presente en el trabajo médico? Desafío que puede desembocar en el encarnizamiento terapéutico.

Esta legítima ambición de curar no debe plegarse a la lógica social imperante, que consiste en excluir la vejez del campo social a través de la medicalización creciente de la relación social respecto de la vida. La medicina, si desea reencontrarse con la integralidad de sus finalidades primeras, debe aceptar pensar su objeto a partir del deseo de rechazar «la mise à mort» (17) en el seno de la práctica médica. En caso alguno se trata de negar la muerte sino de rechazar que la práctica médica desemboque en la muerte de un sujeto que la medicina sabe que no se sanará; se trate de la muerte simbólica de un anciano demente que se le aisla en su estado o de la muerte más real de ciertos ancianos - respecto de finales de vida a los cuales se les nombra púdicamente como «acelerados»- puesto que la existencia de estos altera el orden social y, a veces, el orden institucional del hospital.

La medicina debe también, probablemente, darse como misión recordar a la sociedad que vivir y envejecer permanecen y permanecerán actividades de alto riesgo, con la certeza de la muerte al final del camino. El médico no debe conformarse con la comprobación de que los individuos contemporáneos prefieren las estrategias de fuga respecto de su mortalidad (ya sea bajo la forma del sueño de la salud perfecta, de la valorización de la juventud y de la belleza o de la búsqueda continua de una productividad máxima). La geriatría, los cuidados paliativos, la medicina del dolor, y las prácticas de acompañamiento del final de la vida muestran, por otra parte, que este trabajo se encuentra en curso y que si bien la tarea es dura, ya se encuentra en marcha. El envejecimiento, por lo tanto, representa para la medicina una ocasión de poder precisar de mejor ma- 
nera sus finalidades y sus medios, para evitar, al mismo tiempo, una medicalización no deseable de la sociedad y la politización de la medicina.

Hasta aquí el intento de interpretación de la situación actual de la medicina que nos permite comprender mejor los problemas y los interrogantes planteadas por el hecho de ocuparse médicamente de las personas de edad mayor.

¿Qué hacer entonces para que la medicina mantenga sus finalidades iniciales en el seno de la práctica geriátrica, frente a las presiones políticas y sociales que la empujan a satisfacer objetivos que le son ajenos? Intentemos proponer ciertas referencias que podrían facilitar la orientación de la acción médica respecto del envejecimiento y de sus consecuencias individuales.

\section{Pistas para una orientación más segura de la práctica médica}

La geriatría plantea de manera creciente la necesidad de una colaboración estrecha entre las responsabilidades de médicos, equipos de salud, equipos de instituciones diversas (hospitales, centros de revalidación, casas de reposo y de cuidados), de familias y de pacientes. Para estimular esta responsabilidad, se trata, primeramente, de suscitar la creatividad de los actores y de los sujetos de esta medicina geriátrica, pero también la de los «pacientes» que se inquietan, cada vez más, por los tratamientos que les imponen.

\section{Por una medicina prudente que respete las opciones y la dignidad de cada persona}

Un primer punto de referencia consiste en recordar una evidencia-que los nuevos conocimientos e innovaciones tecnológicas pueden conducir a olvidar- de que el ser humano continuará enfermándose y muriendo. Cada victoria sobre una enfermedad permi- tirá la expresión, probablemente más grande, de nuevas enfermedades (el SIDA es una ilustración trágica, pero también el resurgimiento en ciertas partes del mundo de enfermedades que se consideraban como erradicadas). La muerte puede ser retardada pero nunca suprimida. El dolor y el sufrimiento continuarán siendo parte de la condición humana. Existirán siempre personas que deben ser cuidadas cuando se agoten o lleguen a su límite las capacidades curativas de la medicina. En este contexto, una medicina prudente es más que nunca necesaria. Es decir, una medicina que equilibre su lucha contra la enfermedad a través de la evocación constante de su misión, que es ayudar a vivir en buena salud, en el marco de una existencia forzosamente limitada por la muerte. La medicina puede asegurar esta misión sólo con la plena colaboración de la persona que se confía a ella y en el respeto singular de esta persona, cualquiera sea su edad y su estado.

\section{Por el respeto de la integridad del viejo/a}

El respeto por el anciano enfermo exige probablemente una aproximación más especifíca que el enfermo «habitual». El consentimiento y la información permanecen como nociones centrales, pero pueden ser reinterpretadas a la luz de la noción de sinceridad -más global y más adaptada a la situación geriátrica- que la noción de verdad, más conforme a lo que exige el respeto de la dignidad y de la experiencia de vida concentrada en una persona. Sólo esta sinceridad puede permitir a la persona implicada evaluar mejor, en función de acontecimientos anteriores de su vida y de su «esperanza», la pertinencia de eventuales proposiciones de cuidados. «La sinceridad es lo que debemos al viejo, como forma apropiada de expresarle respeto» (18).

En cuanto a la noción de autonomía y justificación del consentimiento informado, es 
necesario constatar que se encuentra poco asegurada en las situaciones geriátricas, menos aun que en la práctica médica habitual. Consentimiento informado y autonomía de la decisión suponen un paciente perfectamente capaz de comprender las informaciones sobre el diagnóstico y el pronóstico, así como la propuesta de tratamiento, para poder optar, incluyendo la posibilidad de rechazo de todo tratamiento. Esta opción reposa en los valores del paciente y en su visión personal de lo que es bueno para él, en particular, sobre la capacidad del paciente para articular sus valores a la opción de un tratamiento. Pero, en este período de la vida estos valores y esta visión se encuentran a menudo en un proceso de evolución, en la perspectiva del final que se aproxima.

La responsabilidad de los terapeutas no es sólo de entregar la información de forma transparente, con el fin de obtener un consentimiento válido del paciente, sino también, la de asegurar la existencia de procesos e intervenciones que garanticen la significación de la vida de la persona en el momento de su inserción en el circuito de los cuidados. Por ejemplo, una persona enferma de edad mayor no es sólo un paciente, es también padre/madre, amiga y vecina; y los valores, deseos, preferencias y maneras de ser de esta persona se conectan con su inserción en redes relacionales. Es necesario prestar una atención preferente, más que a la cuestión de la autonomía, a la dimensión narrativa de la situación: la experiencia de la persona -relatada por ella o por sus cercanos relevantes-debe poder estar presente en el análisis de la situación y debe, en la medida de lo posible, orientar las decisiones tomadas.

Se trata prioritariamente de preservar la integridad de la historia de vida (del relato de vida) de un paciente: la integridad de la persona, la integridad de lo que esta persona es y lo que su vida significa. Una decisión debería tomarse a través del conocimiento, tan completo como sea posible, de la histo- ria de la persona entendida como un proceso, para ayudar a anticipar lo que debería acontecer, anticipar lo deseable y apoyarse en ello para tomar una decisión. Tal perspectiva implica el respeto de la autonomía, más aún facilita la autonomía, pero, hablando de manera estricta, la sobrepasa. No se trata solamente de basarse en las preferencias conocidas o expresadas por una persona «incapaz» o «incompetente», sino que asegurarse que toda decisión se tomará en la perspectiva del estilo de vida y de los valores de esta persona en una situación determinada. En los hechos, es importante encontrar la mejor manera de integrar un cambio (a veces dramático) en la continuidad de una historia personal, más que evaluar racionalmente las «preferencias» del momento de un «individuo autónomo» que adoptaría sus opciones en consciencia.

El respeto de esta integridad parecer ser una obligación moral que busca «ayudar al paciente a encontrar nuevas formas de significación y a preservar su propia personalidad, tanto tiempo como sea posible» (19).

\section{Por una clínica geriátrica}

El camino médico en materia de geriatría, mucho más que en otros lugares, se encuentra a menudo conducido a perseguir objetivos múltiples, que pueden ser excluyentes los unos de los otros o complementarios: «curar la patología, tratar la deficiencia, restablecer la función, compensar la perdida, aliviar los síntomas, acompañar en el sufrimiento o durante la etapa de la muerte» (20). La mayor dificultad, desde el punto de vista médico, consiste en encontrar, caso a caso, el justo equilibrio en «el hacerse cargo», garantizando que el exceso de cuidados será evitado, absteniéndose, al mismo tiempo, de actos o de tratamientos inútiles y de cuidados desproporcionados.

Por lo anterior, la geriatría aparece como una de las disciplinas donde el desarrollo de una «aproximación del paciente» por 
consenso ${ }^{\text {iii }}$ se torna más difícil que en otros sectores de la medicina (¿qué consenso obtener, por ejemplo, sobre la evaluación de la competencia mental de una persona de edad mayor?) y donde la inteligencia, la formación y la experiencia de los médicos puede ser revalorizada.

Preocuparse en forma competente de los ancianos en el hospital se argumenta a través de la re-legitimación de una cierta experiencia clínica, también de una cierta modestia, así como de la preocupación primordial por la fragilidad de la persona enferma. Hacerse cargo del paciente geriátrico constituye, de esta manera, una excelente ilustración de la prudencia clínica necesaria en toda evaluación de una situación singular: obtener de forma óptima informaciones diagnósticas o pronósticas pertinentes a través de la utilización de medios de investigación con un mínimo de inconvenientes, donde el diálogo tiene un lugar privilegiado, al menos, cuando es posible. Pero incluso si no es posible, dicha situación no impide una participación en la toma de decisión y en la consideración del punto de vista del enfermo sobre su propia situación, cualquiera sea el diagnóstico de tipo psiquiátrico, el retraso mental o la demencia.

La geriatría se caracteriza por una «gran capacidad de adaptación de los profesionales» (21), por la diversidad y la gran heterogeneidad de los distintos segmentos de edad avanzada, por la diversidad de las patologías, por el hecho también de que «...imprime su marca en la expresión clínica de los sínto-

iii Ya sea bajo la forma de una «Evidence-Based Medicine» cuyo principio consiste, para «asegurarse del tratamiento de un enfermo, de examinar lo que dice la literatura en un caso parecido, después de aplicarlo al caso particular» (Chabot, 1998: 531), ya sea bajo la forma de «Recomedaciones para la práctica clínica». Una cierta manera de hacer medicina tiende a dar una gran importancia a esta EBM, en particular, para responder a la pregunta sobre la mejor repartición de recursos. Ver, por ejemplo, en esta perspectiva, Culpepper, Gilbert (1999). mas, sobre el ambiente psico-relacional y sobre la manipulación de las terapias» (21).

En geriatría, particularmente, se explicita lo que es razonable hacer desde la perspectiva de un compromiso prudente con los cuidados de salud. Con todo, es sabido que la geriatría sigue siendo el pariente pobre de la medicina interna, a lo menos en Francia, y que pocos medios son consagrados a la formación y a la investigación en geriatría (por ejemplo, para la menopausia, la osteoporosis, la hipertensión, la diabetes, el funcionamiento cerebral). La importancia de la geriatría debería ser evidente en el contexto de una sociedad que envejece. Sin duda que es necesario ver en esta negligencia hacia la geriatría una confirmación del diagnóstico propuesto sobre la marginalización de la vejez en nuestra sociedad. De hecho, los viejos se cuentan entre los menos aptos para manifestar y presionar en las calles a los poderes.

\section{Por una reflexión ética de la práctica de salud}

En contexto geriátrico las decisiones son frecuentes (por ejemplo, pasar de una estrategia curativa a cuidados de confort y de fin de vida, colocar o no una sonda gástrica, realizar o no transfusiones, abstenerse o no de prescribir antibióticos) y no siempre fáciles de tomar, en particular por la especificidad geriátrica y la importancia que se da a la aproximación global de la persona sanada. Por lo tanto parece importante crear en los equipos implicados las condiciones necesarias a la instauración de discusiones sobre la toma de decisiones. Puesto que si el combate por una práctica digna y respetuosa del anciano en el hospital pasa por el mejoramiento y la mayor frecuencia de una comunicación sincera, así como por el aumento de la competencia médica, parece difícil evitar el desarrollo del diálogo y del debate en el seno de los equipos de terapeutas encargados de las personas ancianas. 
El método y el camino propuesto por la ética clínica puede ayudar. Ésta considera que «en cada situación, es necesario tomar de forma singular la responsabilidad de inventar una respuesta y que cada uno imagine la mejor negociación posible» (22). Es necesario cada vez reinventar de manera creativa una respuesta pertinente que tome en cuenta los imperativos contradictorios de la situación, puesto que «para asumir sus responsabilidades es necesario evaluar los riesgos de cada situación» (22).

Se trata, concretamente, de dar vida a una reflexión ética, a partir de situaciones clínicas identificadas por los actores, como el planteamiento de problemas de difícil solución. Desde un punto de vista ético son consideradas como difíciles aquellas situaciones en donde las opciones no son evidentes para la argumentación, donde las dimensiones de la situación son particularmente duras, donde se busca la orientación más adecuada del «sentido» de la práctica de cuidados que se quiere sostener. Un método de ética clínica ${ }^{\text {iv }}$ busca precisamente intentar trazar un camino sobre el cual, a través de la práctica de cuidados, el profesional y los pacientes puedan mantener el sentido al cual le confieren un valor. Dicho método-camino puede inscribirse en la práctica, como para valorizarla, colocando, al mismo tiempo, en evidencia sus soportes de «moralidad» (cada persona es capaz de conducir su vida moralmente) y abriendo perspectivas por una orientación más adecuada a la finalidad de los cuidados y de la práctica médica.

Las ventajas de este método-camino son múltiples. En primer lugar, permite abandonar la evidencia (en particular, la evidencia de las «buenas prácticas») e interrogarse para comprender la globalidad del contexto en el cual se plantea la pregunta difícil. Es posible pasar de la evidencia de la acción a la prudencia crítica a propósito de la acción, lo

iv Ver Cadoré (1997) para una exposición más detallada al respecto. que evidentemente beneficia a la persona en cuestión. Enseguida, esta reflexión permite clarificar la bruma provisoria de la situación: no se trata de querer suprimir la opacidad de una situación, sino que darse los medios de avanzar prudentemente, puesto que la brumosidad está siempre presente, pero, es menos espesa. Finalmente, esta reflexión permite pasar del discurso «sobre» (sobre la enfermedad, sobre el órgano afectado, sobre los resultados de los análisis) a una interpretación compartida. Se destina tiempo para conversar en conjunto sobre los desafíos planteados por la situación, lo que permite, al mismo tiempo, evitar ahogarse por las dificultades de los profesionales y preocuparse de la realidad prioritaria, es decir, de la persona implicada.

Con el tiempo, la costumbre de discutir y reflexionar en conjunto puede desarrollarse y generar transformaciones tanto personales como colectivas. Nociones como la justicia, el coraje y la honestidad pueden aparecer en la reflexión e influir la vida cotidiana de un equipo.

Por ejemplo: evitar la injusticia en la acogida de las personas (la edad no es un criterio que permita rechazar dar cuidados) al mismo tiempo que la desigualdad entre los pacientes (una sola persona no puede «consumir» una parte importante de los medios disponibles de un servicio si ello no se encuentra plenamente justificado); tener el $\mathrm{co}$ raje de resistir a las presiones familiares y de los próximos, ya sea para «acelerar las cosas» en fin de vida, o al contrario «para intentarlo todo», a pesar de que médicamente consista en un tratamiento manifiestamente inadecuado; tener la honestidad de decir que no es posible hacer nada por esta persona frente al temor de los cercanos de «un abandono» o frente a la repugnancia de ciertos médicos de reflexionar sobre las razones profundas que los conducen a desear detener tal tipo de tratamiento, temor y repugnancia que puede reforzarse por la ausencia de comuni- 
cación en el seno del equipo.

Una medicina prudente, el respeto de la integridad del anciano, una clínica geriátrica, una reflexión de ética clínica pueden contribuir, como referencias, a atenuar la soledad, la impresión de inutilidad -a menudo presente en los enfermos de edad mayor- para favorecer que se mantenga la expresión de la personalidad y de la calidad de vida de la persona adulta; para sostener, en la medida de lo posible, el sentido de la existencia de la persona de edad mayor.

\section{Referencias}

1. Gucher C. Chronique d'une mort programmée. JALMAV 2000; 60: 9-13.

2. Dayez B., Carte Blanche. Le Soir. Bélgica: 18-11-1999.

3. Soubeyrand J., Le bogue des petis vieux. Liberation. 24-01-2000.

4. Krémer P., Le Monde, 5-01-2000, p. 8.

5. Poncin J.. La vieillesse n'est pas un naufrage. Le Soir. Bélgica: 18 de enero 2000, p. 12.

6. Haut Comité de la Sante Publique. La Santé en France: 1994-1998. Paris: La Documentation française; 1998.

7. Miller TH, Bielen FR, Dani M, Boitte P. Echecs de la prise en charge de la personne âgee. Solutions possibles: expérience d'une équipe médicale. Inédit. Ottignies: Clinique Saint-Pierre. 1998. p. 7. [On Line] Soliciter a Dr. Miller: mul3@skynet.be

8. Deplanque R. Management et bienveillance dans les soins: quelle stratégie. Gestions Hospitalières 1999; 384: 179-85.

9. Baulon A. Insistance ou abstention thérapeutique en gériatrie. La lettre de l'Espace éthique - AP -HP 2000; 11:37-40.

10. Tong R. Towards a Just: courageous and Honest resolutions of the Futility Debate. The Journal of Medicine and Philosophy 1995; 20: $165-89$.

11. Sebag-Lanoe R. La décisionface aux situations du grand âge. La lettre de l'Espace éthique - AP - HP 2000; 11: 33-5.

12. Richard MS. La personne âgée en institution. Dolentium Hominum 1999; 40(1): 79-82.
13. Wils J. Refus de soin et conflit de conscience. La lettre de l'Espace éthique-AP-HP 2000; 11:36-7.

14. Nordin I. The Limits of Medical Practice. Theoretical Medicine and Bioethics 1999; 20: 105-123.

15. The Hastings Center. The goals of Medicine: setting new priorities. Hastings Center Report; Special Suplemment 1996; S1-S27.

16. Laplantine F. Anthropologie de la maladie. París: Payot; 1986.

17. Pontalis JB. Une idée incurable. In: Pontalis JB, (ed). Perdre de vue. París: Folio/ Essais; 1999, p. 79-90.

18. Kuczewski MG. Ethics in long-term care: are the principles different. Theoretical of Clinical Ethics 1999; 20: 15-29.

19. Holstein MB. Ethics and Alzheimer's Disease: Widening the lens. The Journal of Clinical Ethics 1998; 9(1): 9-22.

20. Voyer G. Chapitre 50: Ethique clinique. In: Arcand- Herbert, (ed). Précis practique de gériatrie. Montreal- Québec: EdisemMalouin; 1998. p.677-87.

21. Sebag-Lanoe R. Les soins palliatifs en gériatrie. Actualité et dossier en santé publique 1999; 28: 46-50.

22. Derrida J. Sur Parole: instantanés philosophiques. Paris: Edittions de 1'Aube; 1999.

\section{Bibliografía}

Boitte P. Ethique, justice et santé: allocation des ressorsces en soins dans une population vieillissante. Montréal: Artel; 1999.

Boitte P. La valeur de la vieillesse: enjeux éthique et politiques. Info Nursing 1996; 63:24-8.

Boitte P. Pour une éthique de la santé publique dans une société vieillisante. Montreal: Artel; 1997.

Cadore B. L'éthique clinique comme philosophie contextuelle. Montreal: Artel; 1997.

Chabot JM. Evidence - Based Medicine (I). La revue du Practicien 1998; 48: 531-2.

Chabot JM. Evidence - Based Medicine (II). 
La revue du Practicien 1998; 48: 651-2.

Comité Consultatif National D’Etique. Rapport sur le vieillissement. Cahiers $d u$ CCNE 1999; 18: 3-24.

Culpepper L, Gilbert TH. Evidence and ethics. The Lancet 1999; 353: 829-31.
Henrard JC. Santé publique et grand âge. Actualité et dossier en santé publique 1997; 20: 2-12

Henrard JC, Ankri J. Grand âge et santé publique. Rennes: Editions l'Ecole Nationale de Santé Publique; 1999. 